\title{
Appendix A: Glossary
}

Alignment

An alignment is a comparison of two or more sequences which places the sequences in a matrix, indicating corresponding segments by placing them in the same row, with missing segments being represented by a gap symbol (usually a '-').

Alignment site

A column of an alignment (term adopted from molecular biology). See phonetic alignment analysis.

Basic vocabulary

Referring to concepts that are assumed to occur in all human languages and to be more resistant to replacement than other parts of the vocabulary.

\section{Cognacy}

A relation between two word forms. The relation holds when the two words go back to a common ancestor. Words that share this relationship are called cognate or etymologically related. When talking about "cognates", this usually excludes those words related by borrowing events.

Colexification

Two different concepts that are expressed by the same word are said to colexify.

Compound words 
Words that are formed by combining two other words, like correspondence pattern being composed from the words correspondence and pattern.

\section{Correspondence patterns}

Also sound correspondence patterns. Due to the regularity of sound change (see below), words that share the same sound in one language often also share the same (possibly different) sound in another language if they are cognate with the respective words in that language. These regularities are called correspondence patterns.

\section{Cross-semantic cognates}

Cognate words that have a different meaning due to semantic change.

\section{Morpheme}

Smallest part of a word that corresponds to a meaning or function of its own, usually by occurring in other words as well. It differs from a phonestheme in so far as all morphemes of a word taken together build the whole word, but the parts of a word not belonging to a given phonestheme consist not necessarily of morphemes or phonesthemes themselves.

Orthography profile

A replacement table used to automatically convert data from one transcription system into another (e.g., into IPA) and to segment it into units (e.g., phonemes, diphthongs).

\section{Phonetic alignment analysis}

The comparison of sequences, e.g., of words suspected to be related. The words are therefore put into a matrix in such a way that corresponding segments appear in the 
same column, while placeholder symbols are used to represent those cases where a corresponding segment is lacking.

\section{Strict cognates}

Related words that differ only by regular sound change. This means that they go back to exactly the same word form and that no borrowing event was involved in the history of these words since their common ancestor.

Reflex

The descendant of a given ancestral form. Reflex typically refers to a word form, but one can also find the term reflex sound in the literature. 


\section{Appendix B: Tutorial}

This tutorial supplements the study "Computer-Assisted Language Comparison: State of the Art". In this tutorial, we explain in detail how our workflow can be tested and applied. The workflow consists of several Python libraries that interact; one producing the data that can be used by the other. Since the data is available in different stages, each stage allows us to intervene by correcting errors manually that were made by the automated approach.

For users who are interested in testing our workflow on their local machine or further applying it in their own research, some basic knowledge of the Python programming language and the command-line will be required. All the software offered here is available in the form of free software. For more information on LingPy, the main programming library used here, we recommend that users check the tutorial ${ }^{1}$ accompanying the study "Sequence comparison in computational historical linguistics" ${ }^{2}$ by List et al. (2018) [1].

\section{Code Ocean Capsule}

In order to facilitate it for users to quickly test our workflows without installing the software, we have set up a Code Ocean Capsule which users can use to run the code remotely. Code Ocean is an open access platform which enables researchers to reproduce their or others' experiments. For a detailed introduction to the Code Ocean platform ${ }^{3}$, please refer to the website. To see how our experiments can be run from within the Code Ocean Capsule, follow these steps:

a) Navigate to the capsule: https://codeocean.com/capsule/8178287/tree/v2

b) Press the Re-Run button to reproduce the results.

c) View the progression in the Terminal panel.

d) Download all results and unzip the .zip file for further inspection on EDICTOR. 


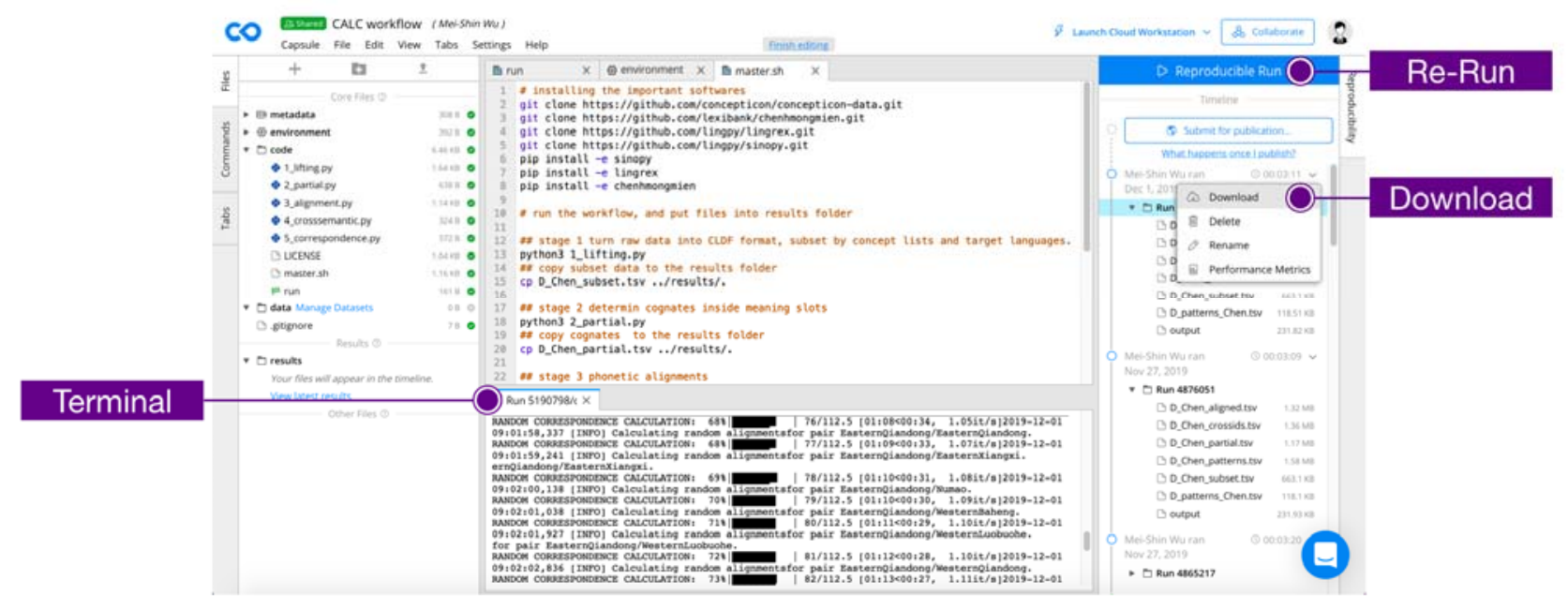

The following files can be found in the downloaded file:

\begin{tabular}{lll}
\hline File & Stage & Section \\
\hline D_Chen_subset.tsv & From Raw Data to Tokenized Data & 3.1 \\
\hline D_Chen_partial.tsv & From Tokenized Data to Cognate Sets & 3.2 \\
\hline D_Chen_aligned.tsv & From Cognate Sets to Alignments & 3.3 \\
\hline D_Chen_crossids.tsv & From Alignments to Cross-Semantic Cognates & 3.4 \\
\hline D_Chen_patterns.tsv & $\begin{array}{l}\text { From Cross-Semantic Cognates to Sound } \\
\text { Correspondence }\end{array}$ & 3.5 \\
\hline D_Chen_distance.dst & Validation & $4.2,4.3$ \\
\hline D_Chen_tree.tre & Validation & $4.2,4.3$ \\
\hline
\end{tabular}

\section{Installation Instructions}

We assume that users who are interested in running the workflow on their local machine are familiar with the essentials of command-line operations and system administration on either Unix-like systems (such as Linux and macOS) or Windows systems. Also, users should have Python ${ }^{4}$ installed, including the package manager pip. Additionally, the version control system ${ }^{5}$ git will be required. We strongly encourage users to run this code in a virtual environment. A

$4 \quad$ https://www.python.org/, Version 3.5 or higher

$5 \quad$ https://git-scm.com/ 
virtual environment is a practical solution for creating independent configurations for testing and experimenting, with no interference on the system-wide installation and without requiring complex virtualization or containerization solutions. The Python Packaging User Guide ${ }^{6}$ gives clear instructions on setting up a virtual environment on Windows, Linux and macOS.

We start by installing the dependencies from the command-line. In order to do so, we first download the code that we will use with the help of git.

\$ git clone https://github.com/lingpy/workflow-paper.git

\$cd workflow-paper

Now that we have done this, we can install all the packages we will need with the help of pip.

\$ pip install $-r$ requirements.txt

Now that this has been done, we need to configure the access to reference catalogs, such as Concepticon ${ }^{7}$ and $\mathrm{CLTS}^{8}$ in order to make sure that they can be accessed readily by the code. This can be done with the help of the catconfig argument submitted with the cldfbench package which organizes the linguistic datasets.

\$ cldfbench catconfig

You will be prompted to ask if you want to clone actual versions of Concepticon, Glottolog, and CLTS, and the easiest way to deal with this is to agree and type "y" in all cases.

\section{Getting Started}

There are two basic ways in which you can run our workflow:

1. You can run it by downloading a set of Python scripts and running them directly on your computer.

2. You can use the cldfbench package to run the commands via the command-line, without downloading the data directly. 
The advantage of solution 2 is that you do not have to download extra data, since we have integrated the code directly in the lexibank version of the dataset of Hmong Mien languages by Chén (2012) [2]. Once this dataset has been installed (and this is the first package we have installed in the previous section as part of all dependencies needed), you can type commands on your command-line, and the code will be carried out. The disadvantage is that the code example itself is not that easy to process for people less experienced with Python. For this reason, we will only note the commands in each of the steps we discuss in the following, and not explain them in more detail.

\subsection{From Raw Data to Tokenized Data}

The first script essentially loads the data from the repository and creates a wordlist that contains a subselection of all the data that was used. Some aspects of the more difficult "lifting" of data have already been done and distributed along with the original data package ${ }^{9}$, which specifically also contains the orthography profile in the file etc/orthography.tsv and can be automatically applied with help of the cldfbench package.

\$ cldfbench lexibank. makecldf chenhmongmien

But since the data is available in the form of a cldf package with the original orthography already tokenized to the formats we need, you can also skip this step and convert the data to the wordlist format required by the lingpy package.

\$ python 1_select.py

If you want to test the version from the CLDF-repository directly with cldfbench, you can type:

\$ cldfbench chenhmongmien.wf_select.

This will select a part of the languages and a part of the concepts, as indicated in the main study and write them to a file $D_{-}$Chen_subsets.tsv. Additionally, you will see some statistics on the terminal, specifically a table indicating the coverage for each language. If you want to select all languages, and not just a subset, type:

9

https://github.com/lexibank/chenhmongmien 
\$ python 1_select.py all

The output A_Chen_subset.tsv is generated due to the argument all is used. Once the argument all is used in the first stage, it has to be added to the rest of the stages to ensure that the workflows process the correct files.

\begin{tabular}{lcr}
\hline Doculect & Words & Coverage \\
\hline Bana & 502 & 1.00 \\
\hline BiaoMin & 488 & 0.97 \\
\hline CentralGuizhouChuanqiandian & 454 & 0.90 \\
\hline Chuanqiandian & 501 & 1.00 \\
\hline EasternBahen & 492 & 0.98 \\
\hline EasternLuobuohe & 499 & 0.99 \\
\hline EasternQiandong & 442 & 0.88 \\
\hline EasternXiangxi & 492 & 0.98 \\
\hline Numao & 490 & 0.98 \\
\hline WesternBaheng & 500 & 1.00 \\
\hline WesternLuobuohe & 488 & 0.97 \\
\hline WesternQiandong & 494 & 0.98 \\
\hline WesternXiangxi & 502 & 1.00 \\
\hline Younuo & 500 & 1.00 \\
\hline ZaoMin & 455 & 0.91 \\
\hline
\end{tabular}

Already now you can inspect the data with the help of the EDICTOR tool. In order to do so, open the tool's website at https://digling.org/edictor/ and wait until the page is loaded (note that we recommend to browse EDICTOR in Firefox, but GoogleChrome should also not cause further problems).

The data is in the file D_Chen_subset.tsv. In order to load it to the tool, press the Browse button and select the file. Once this has been done, press the Open the file button to examine the data, as illustrated in the following figure. 


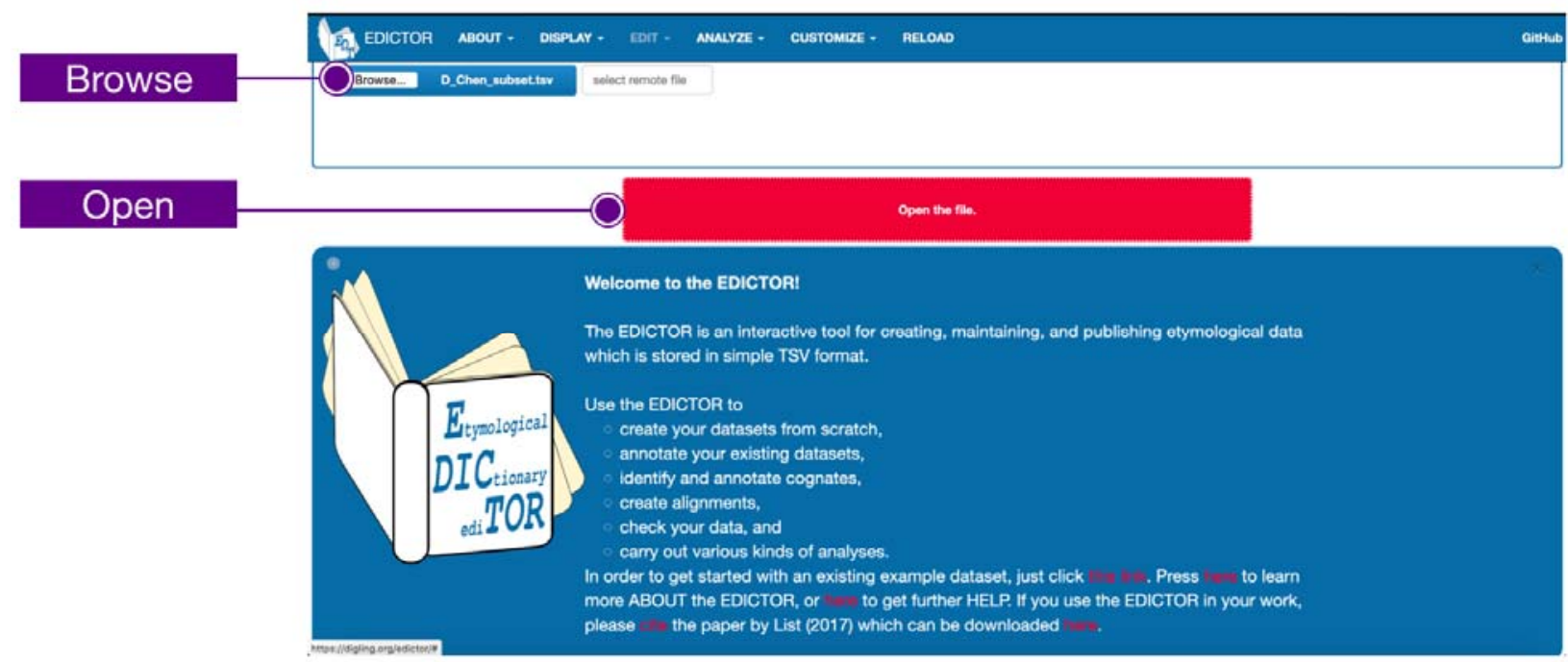

The segmented strings are displayed in the TOKENS column. Press Select Columns to inspect the raw forms and other aspects of the data, as shown in the following figure.

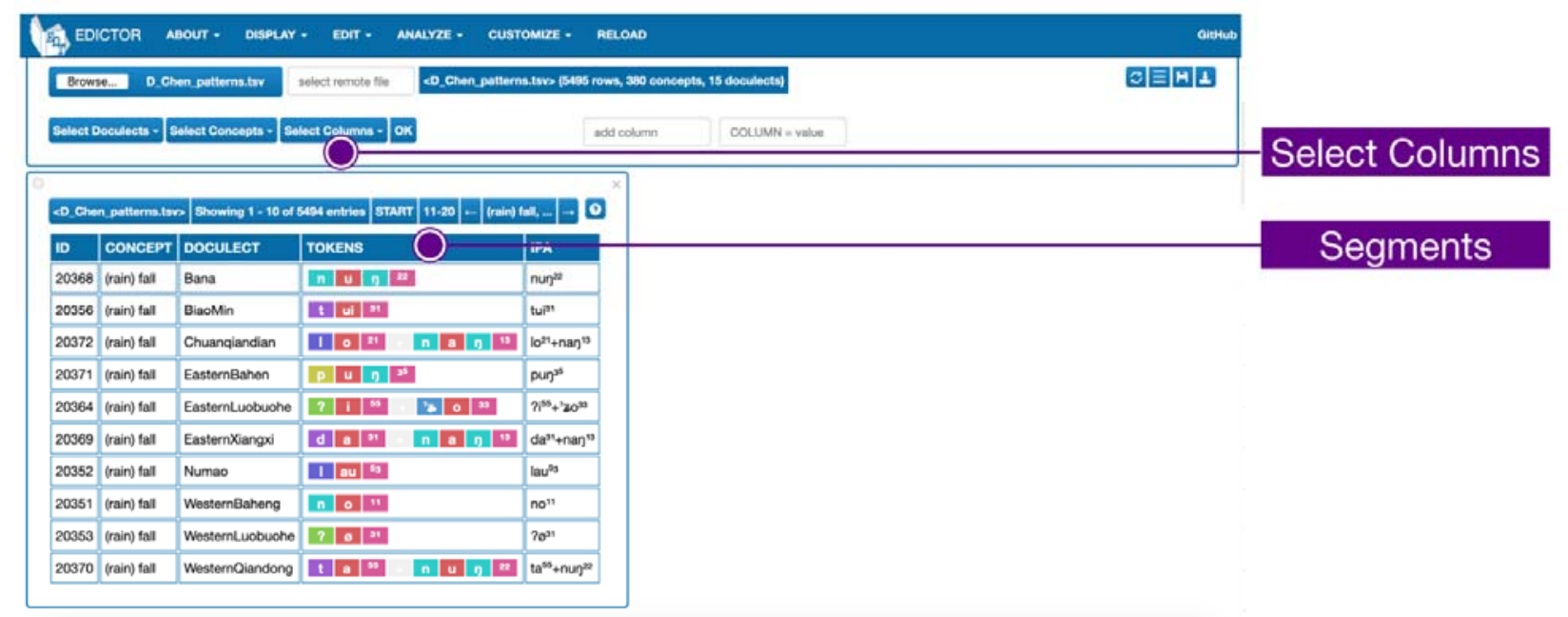

In order to save data to your computer, after you have manually edited them, you need to "download" them. This may be a bit surprising, since effectively, you do not download the data, but since the EDICTOR is working on a browser, it does not have any access to the data on your computer, and download is the only way to communicate with your machine. Thus, in order to save your data and load it to your machine, you first have to press the save icon at the top-right corner in order to store the edited data in the web browser. When now pressing the download icon at the top-right, your browser will either directly download the data and store them in your download folder, or it will ask you to specify a specific file destination. 


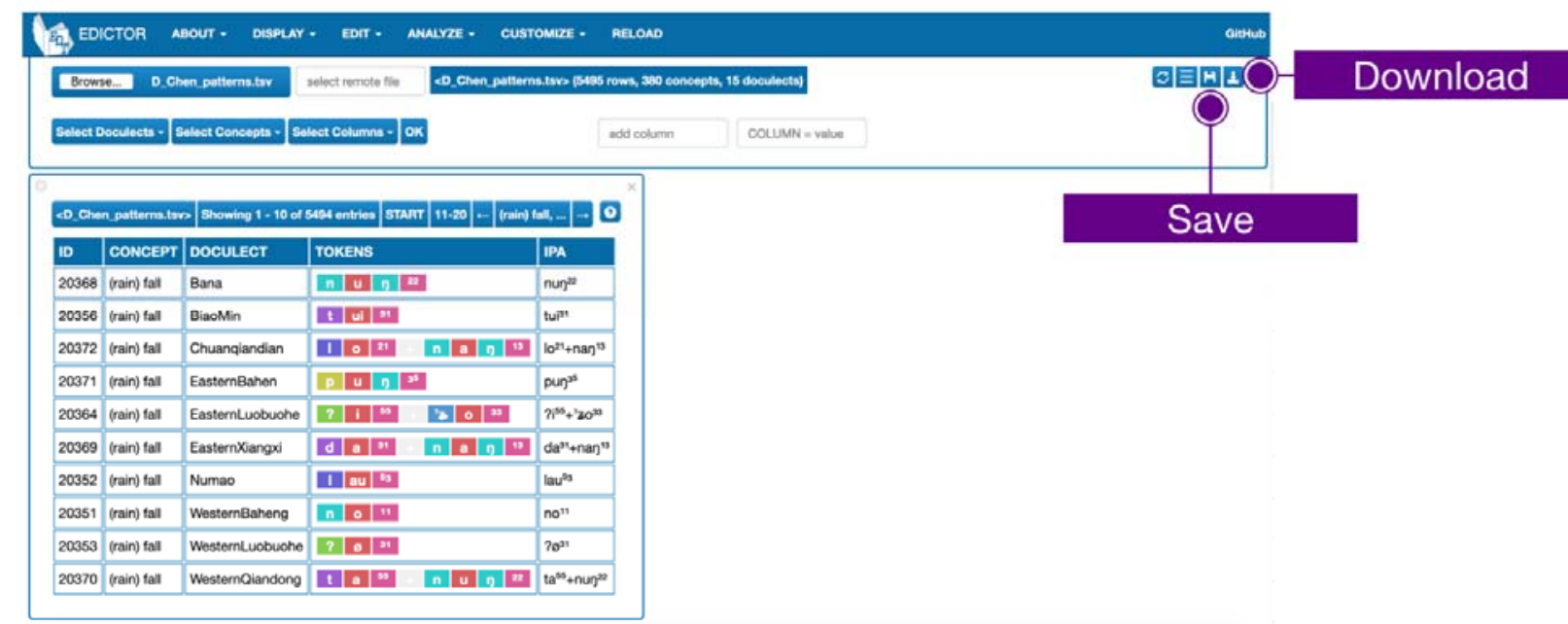

Be careful when editing data in the EDICTOR without saving and downloading them. If you close your browser, all the edits you made will be lost, so you should regularly save and download your data when working with the EDICTOR. As a shortcut, you can also type CONTROL+S to save and CONTROL+E to "export" the data (i.e., to download them).

\subsection{From Tokenized Data to Cognate Sets}

Partial cognate detection is an important task, specifically when working with Southeast Asian language data. The algorithm we use for this task was first proposed in the study "Using Sequence Similarity Networks to Identify Partial Cognates in Multilingual Wordlists" by List et al. (2016) [3], where the algorithm is described in due detail. To illustrate how the algorithm works, we provide an example with four words for 'moon' in the Eastern Baheng, Eastern Qiandong, Bana and Biao Min language varieties. The major steps of the algorithm are the following:

a) Calculate the distances of all morpheme pairs.

b) Create a fully connected network from the distance scores.

c) Filter the network by deleting edges in the following fashion:

A. Two morphemes in the same word should not be linked (see the dashed lines in the following figure).

B. A morpheme in a word should not be linked to two morphemes in another word (see the yellow edges in the figure).

d) Remove the edges with similarity scores below a given threshold. 

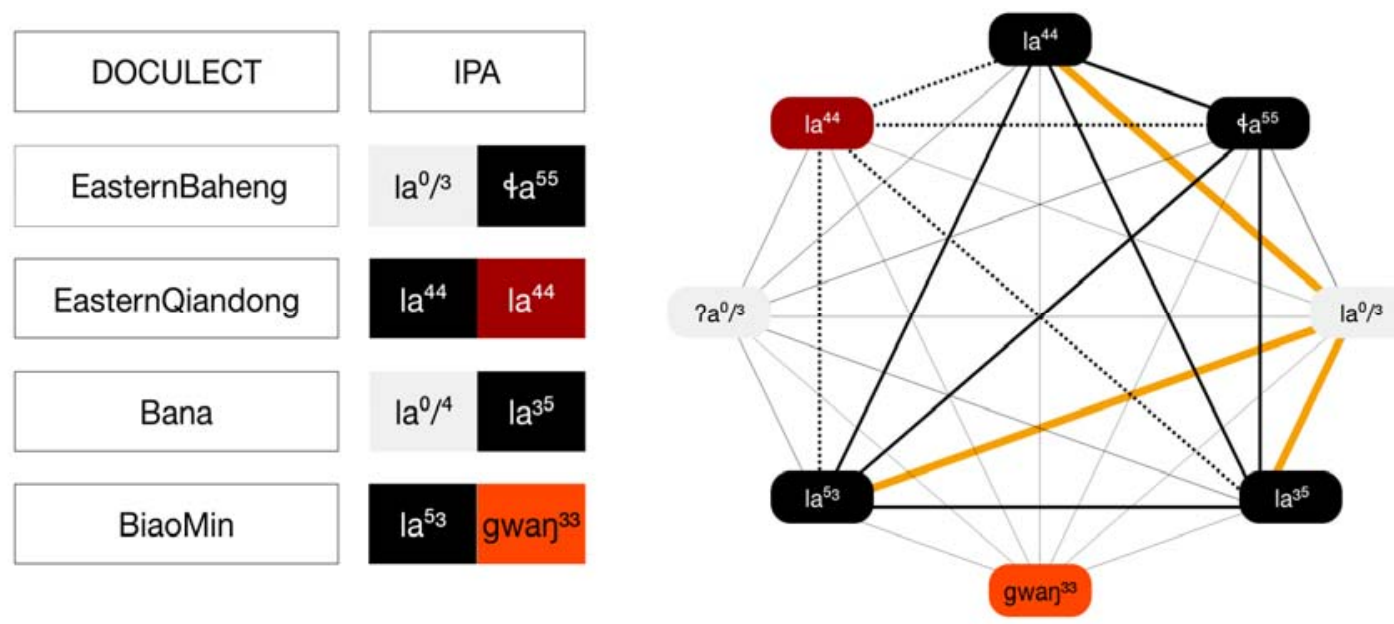

Once this has been done, an algorithm for Community Detection in networks [4] is used to partition the network into "communities", with each community representing one partial cognate set.
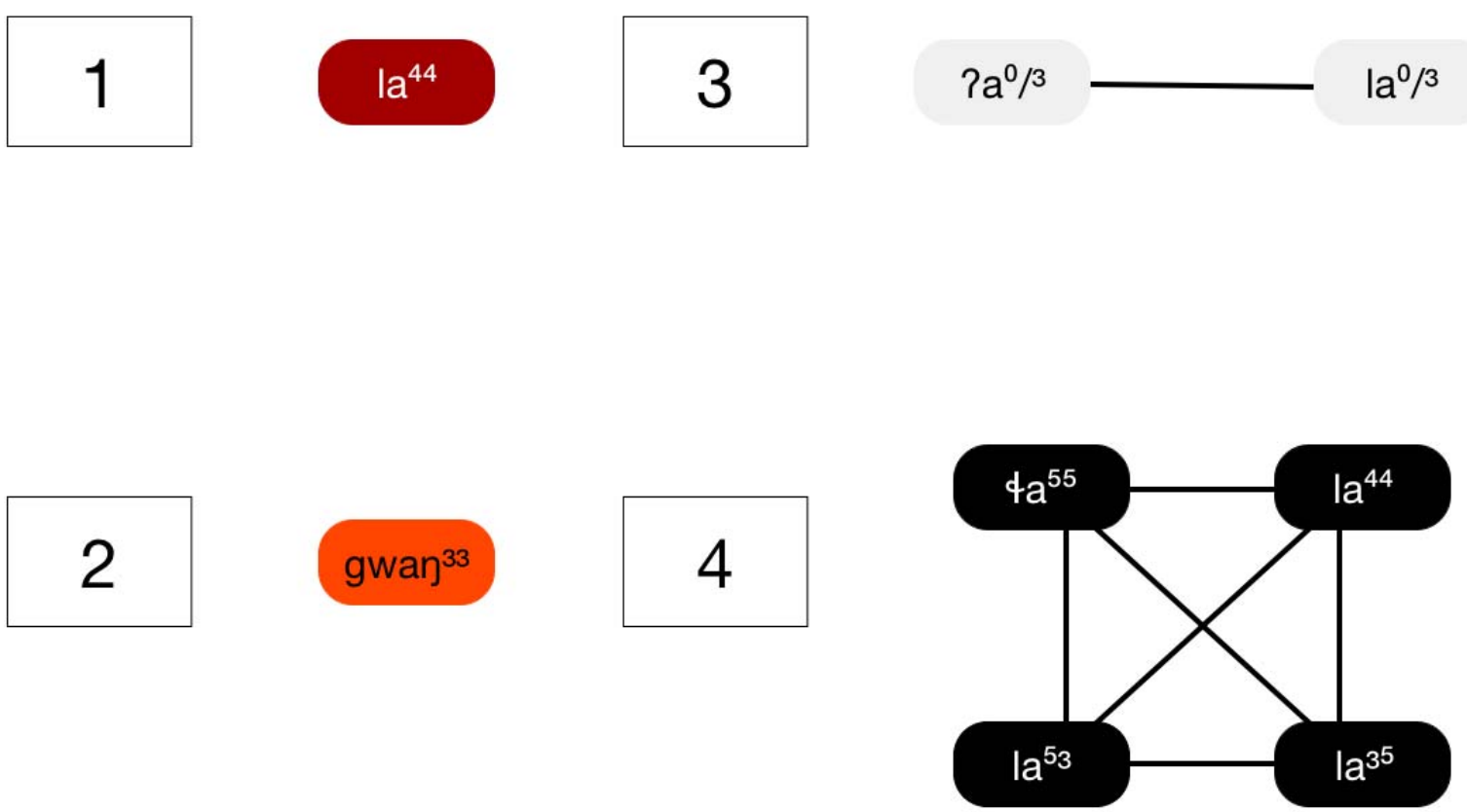

In order to calculate partial cognates, we use the algorithm as provided by the lingpy software package and apply it to our subselection of languages.

\$ python 2_partial.py 
If you want to test the version from the CLDF-repository directly with cldfbench, you can type:

\$ cldfbench chenhmongmien.wf_partial.

This will take some time when you run it the first time. The data can be found in the file D_Chen_partial.tsv. To inspect the data with EDICTOR, load D_Chen_partial.tsv as shown before. Then press DISPLAY to select SETTINGS in the drop-down menu. Select PARTIAL in the Morphology and Colexification Mode entry. Press the Refresh button.

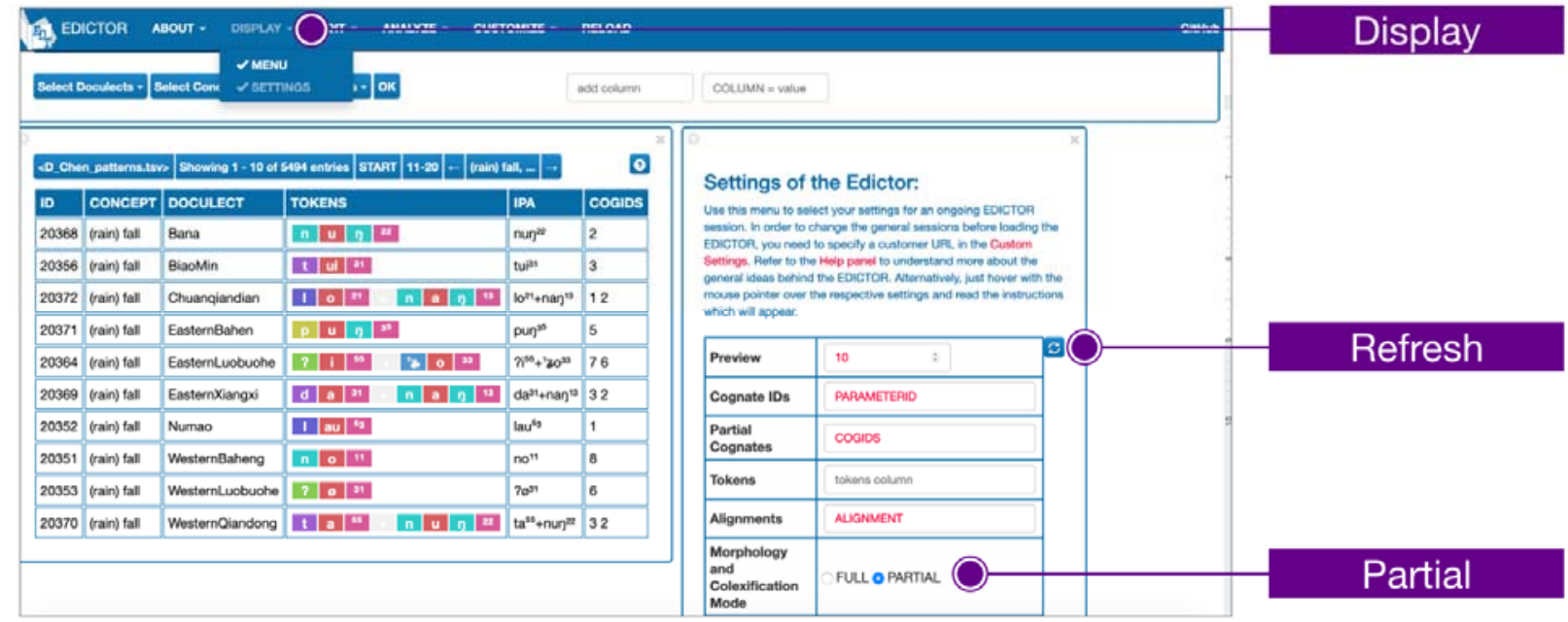

In order to investigate the partial cognates, you need to select the column which stores the identifiers. To do so, press Select Columns and select COGIDS in the drop-down menu.

If you right-click on any number in the "COGIDS" column, a pop-up window will open and show all the cognate sets for a given word form in the form of an alignment. Since we have not yet aligned the data, the alignment will be wrong at this point. 


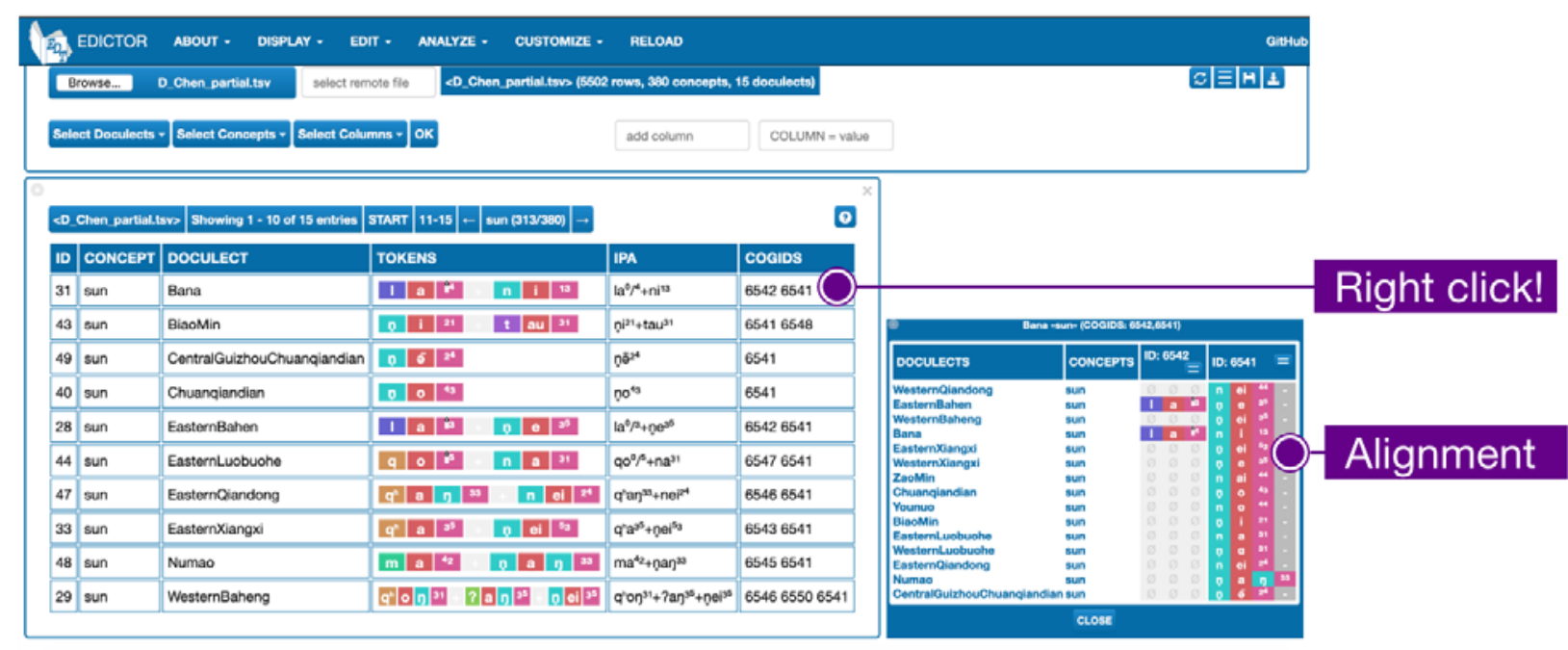

\subsection{From Cognate Sets to Alignments}

To align the data, we use the new procedure for template-based alignment, which is available from the lingrex package which we have installed as one of the requirements of our workflow, and the sinopy package, which helps us to compute syllable templates from all morphemes in the data. Running the code is again straightforward.

\$ python 3_alignment.py

If you want to test the version from the CLDF-repository directly with cldfbench, you can type:

\$ cldfbench chenhmongmien.wf_alignment

The aligned data will be stored in the file D_Chen_aligned.tsv. To inspect the alignments in EDICTOR, load this file and follow the previous steps we mentioned in Section 3.2. In addition to selecting the COGIDS column now, we also select the STRUCTURE column, since this column provides the templates for each morpheme, which we have automatically added to the data with the help of sinopy. 


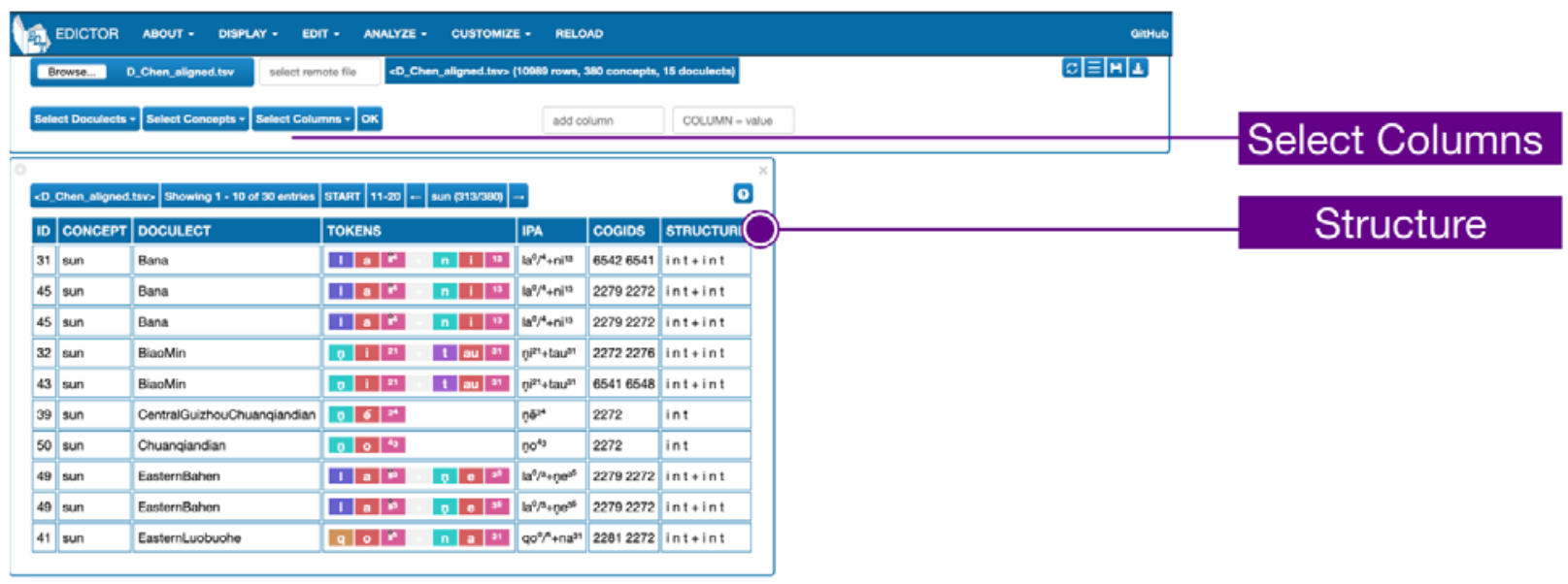

As we already mentioned, if you right-click on any number in the "COGIDS" column, a pop-up window will show the alignment. Click on the = sign to modify the alignment. The modification itself is very straightforward: just click on a sound segment to move it to the right, and click on a gap segment to delete this segment.
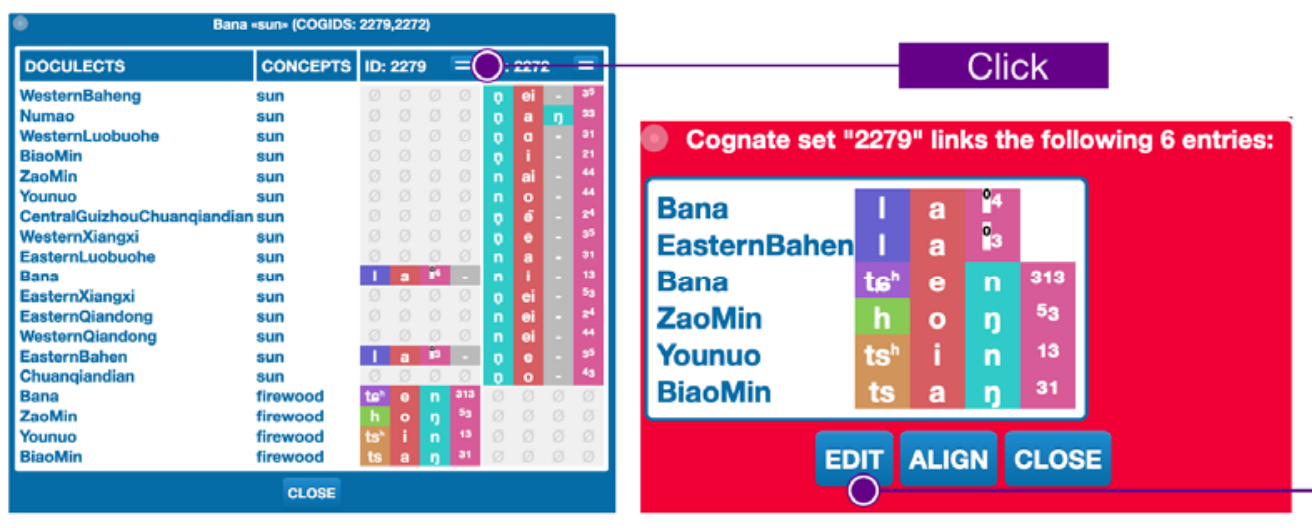

\section{Edit}

\subsection{From Alignments to Cross-Semantic Cognates}

The algorithm for cross-semantic cognate detection as we propose it here is illustrated in more detail in the main study. It is implemented as part of the lingrex package. Again, it is straightforward to run the code.

\$ python 4_crosssemantic.py

If you want to test the version from the CLDF-repository directly with cldfbench, you can type:

\$ cldfbench chenhmongmien.wf_crosssemantic 
The output file is D_Chen_crossids.tsv, and we load it into the EDICTOR tool, just as we did before, but when checking the SETTINGS in the menu this time, we need to specify that the column "CROSSIDS" holds the partial cognates. To do so, just type in CROSSIDS in the text field Partial Cognates in the settings menu and then press the refresh button.

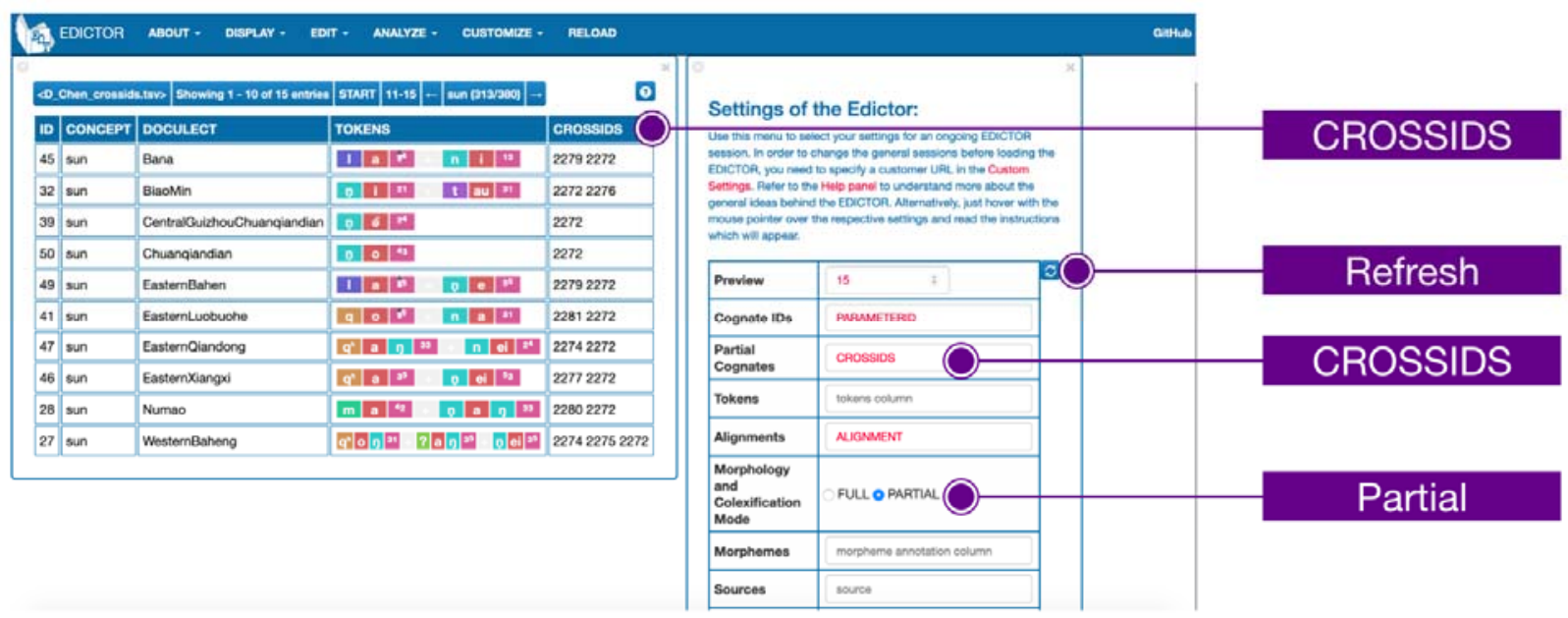

To inspect the distribution of partial cognates, press ANALYZE in the top-level menu and select Cognate sets in the drop-down menu.

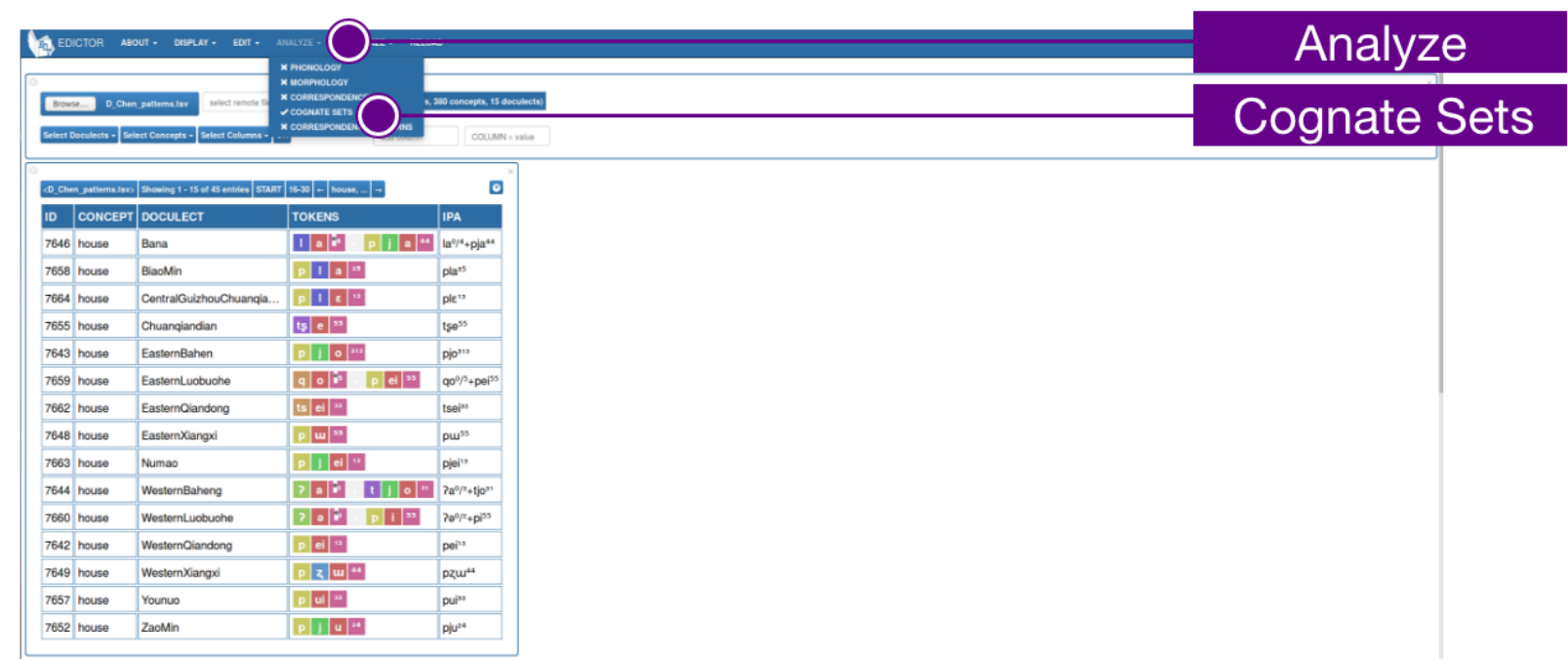

As a result, a new panel will open and show the distribution of all cognate sets across the different language varieties. Pressing the red button with the cognate set identifier on the left will open the alignment. Pressing the yellow buttons with the word identifiers will show you the original morpheme. On the right, in the column CONCEPTS, you will find those cognate sets which are attested for more than one concept as separated by a comma. Clicking on this field will modify the main wordlist panel in such a way that only the selected concepts will appear. 


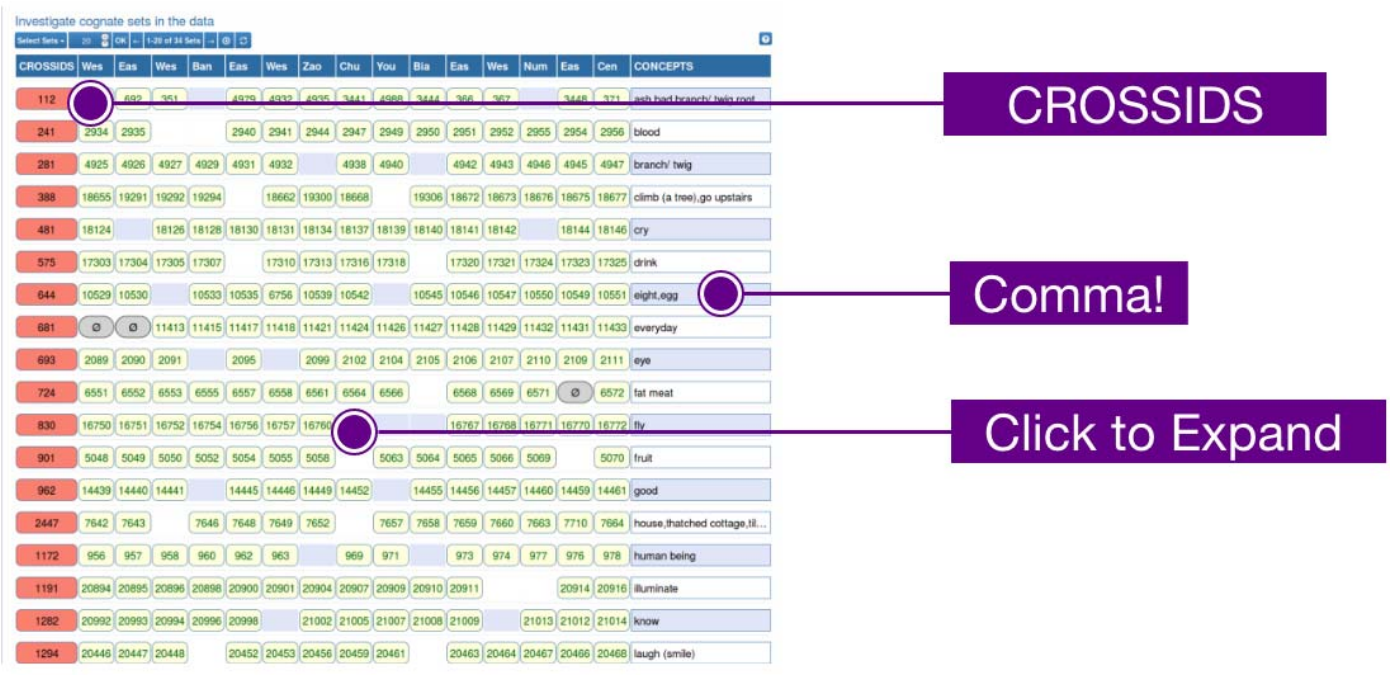

\subsection{From Cross-Semantic Cognates to Sound Correspondence Patterns}

As a final step, we will try to infer the major correspondence patterns in the data, using the algorithm by List (2019) [5] which is available from the lingrex package. Running the code is straightforward, as before.

\$ python 5_correspondence.py

If you want to test the version from the CLDF-repository directly with cldfbench, you can type:

\$ cldfbench chenhmongmien.wf_correspondence

This creates two output files. One, called D_Chen_patterns.tsv, is the file without wordlist that can be loaded by EDICTOR and inspected, and one file contains the patterns that have been inferred alone, called D_patterns_Chen.tsv. In order to inspect the patterns, we recommend using the EDICTOR tool, which requires the same steps that we already applied when loading our cross-semantic cognates. Once this has been done, press the ANALYZE button in the top menu and select CORRESPONDENCE PATTERNS in the drop-down menu.

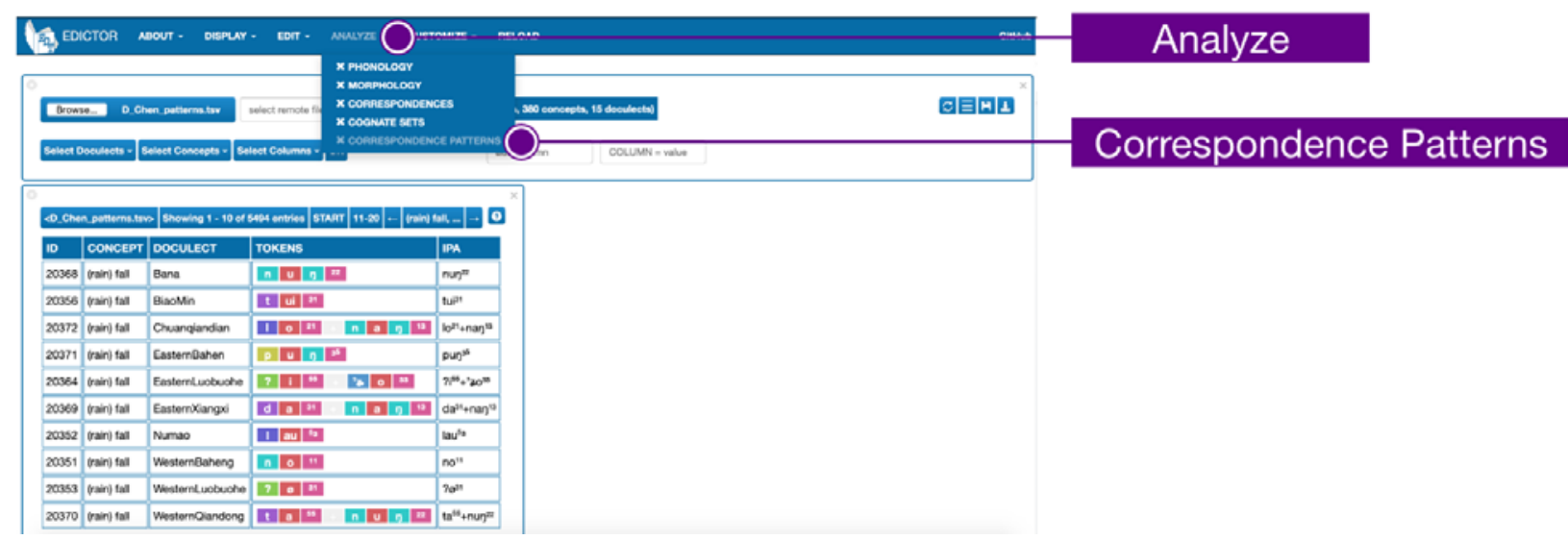


In order to allow for a good display, the Doculect names are all abbreviated. Hovering the mouse cursor on an abbreviation will show you the full name.

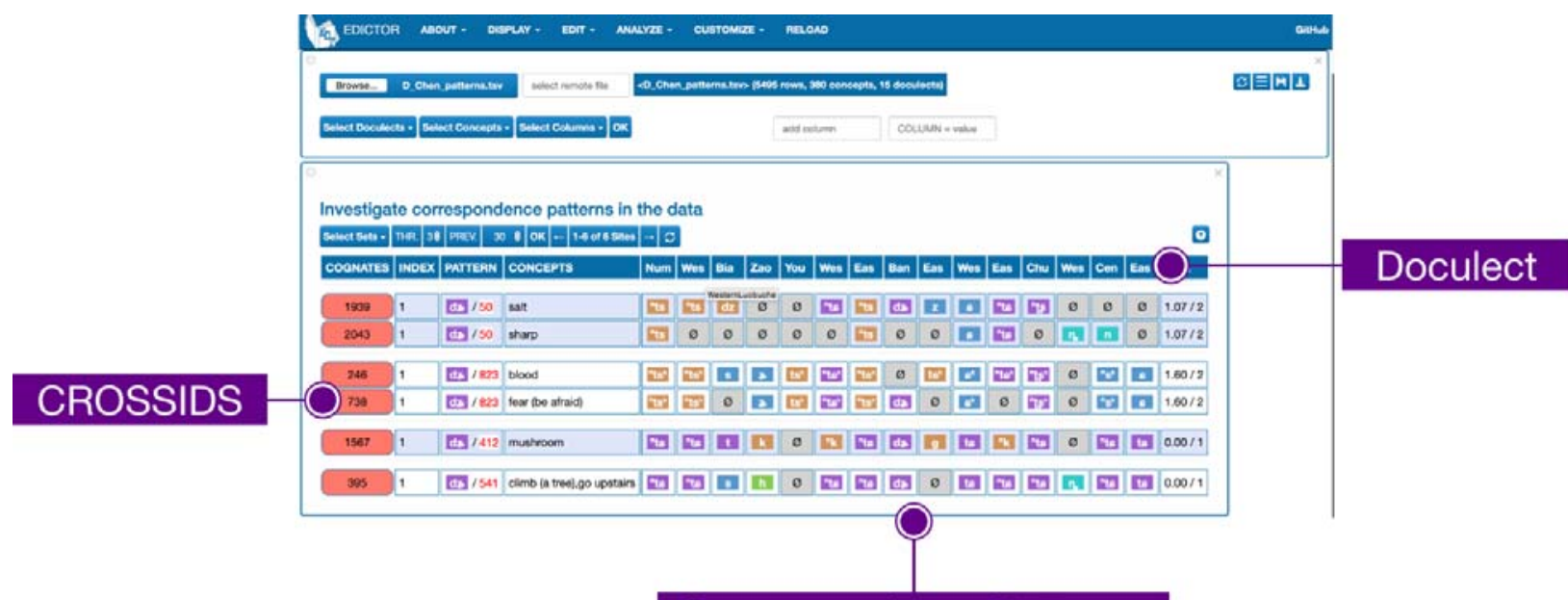

\section{Correspondence Patterns}

Clicking on a cell in the correspondence pattern panel will allow you to see not only the sound in question, but the full morpheme in which this sound occurs.

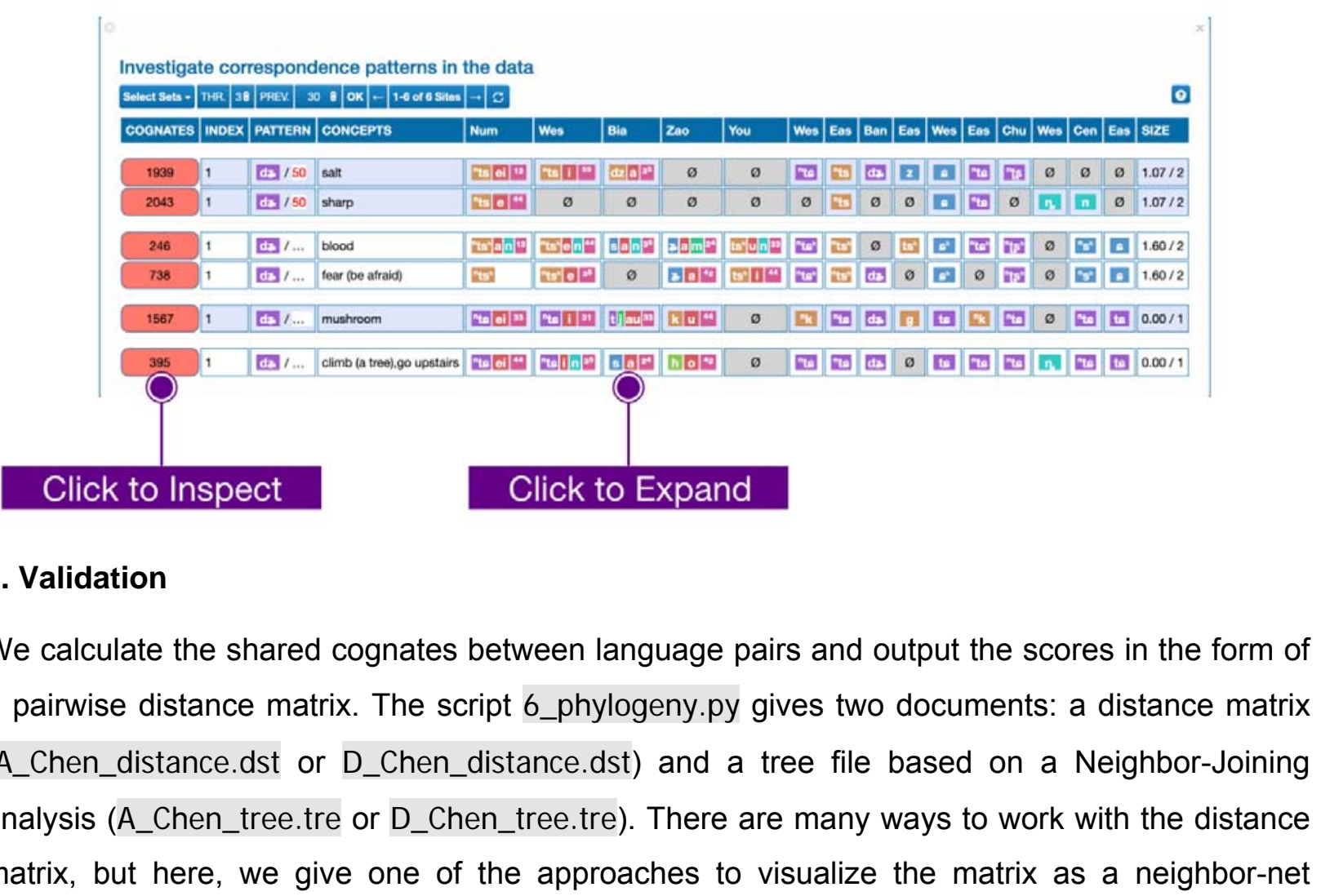


network with the help of SplitsTree. To get started, first make sure to install SplitsTree ${ }^{10}$ [6] and follow the installation instructions. In order to compute the distance matrix with our code, use the command-line (here we compute it for the entire dataset, so we run it with the keyword all).

\$ python 6_phylogeny.py all

To generate a Neighbor-Net from the distance matrix, open the file A_Chen_distance.dst or D_Chen_distance.dst with any plain text editor and start the SplitsTree software. Then click on File and Enter Data, as shown in the image below.

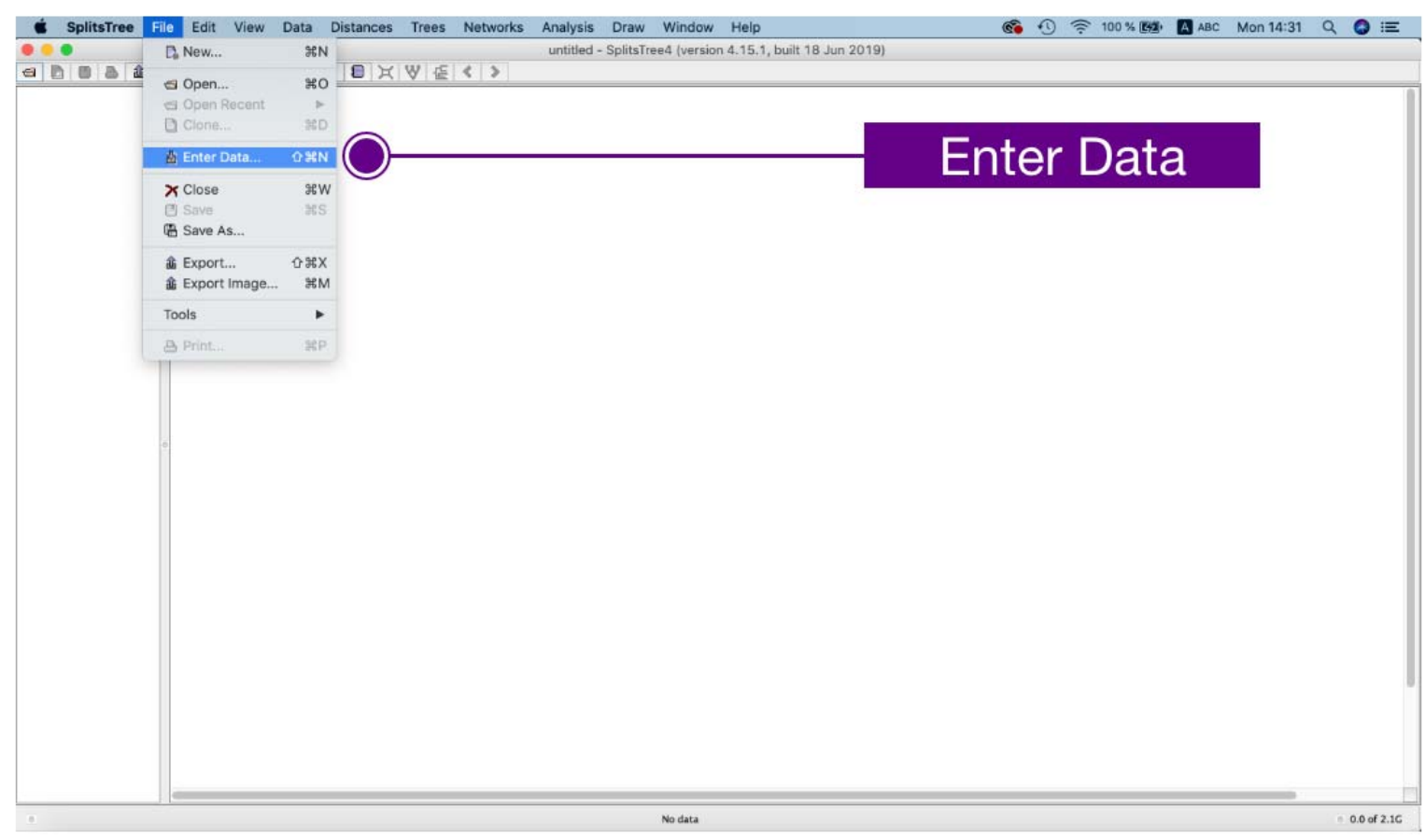

Then copy the distance matrix and paste it into the Enter Data Dialog, and press Execute. 


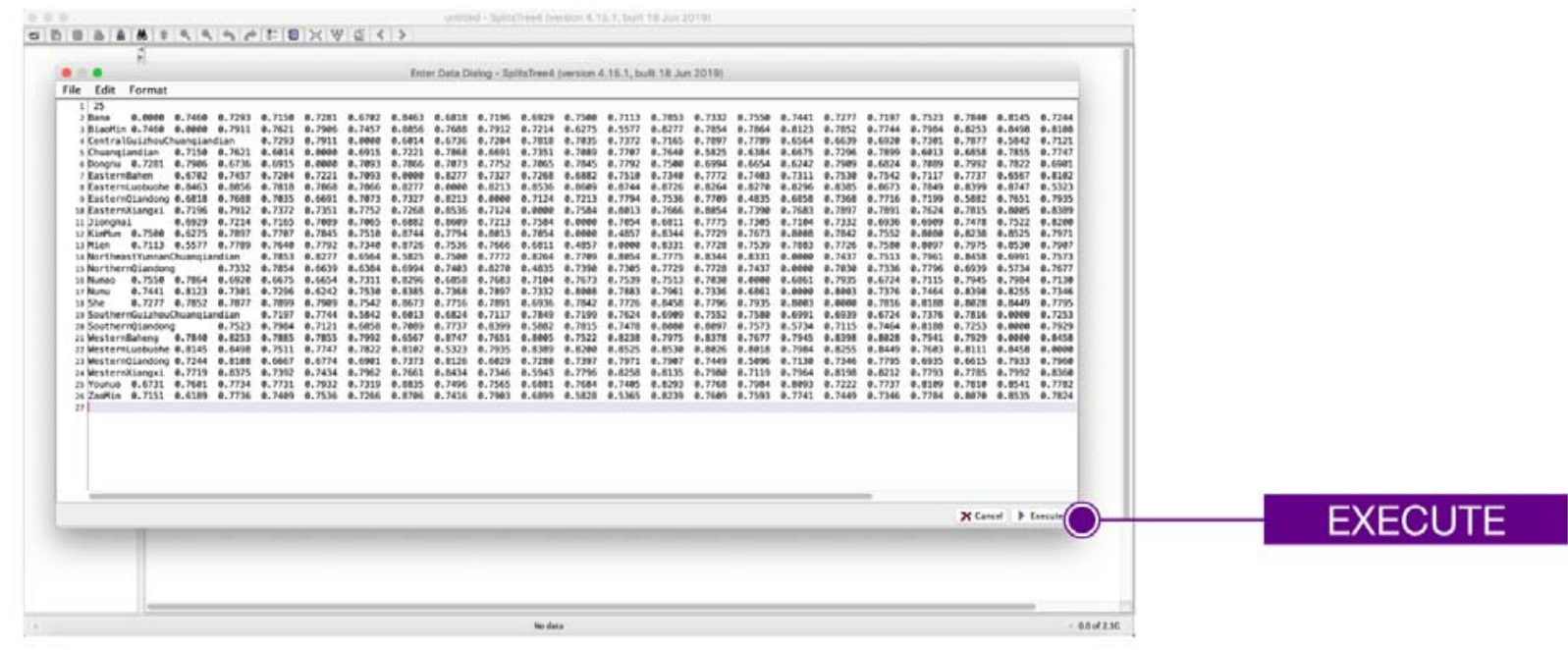

You can now inspect the network. To analyze the data further, you can compute the delta scores, showing the degree of reticulation in the data, by pressing Analysis and then Compute Delta Score, as shown below.

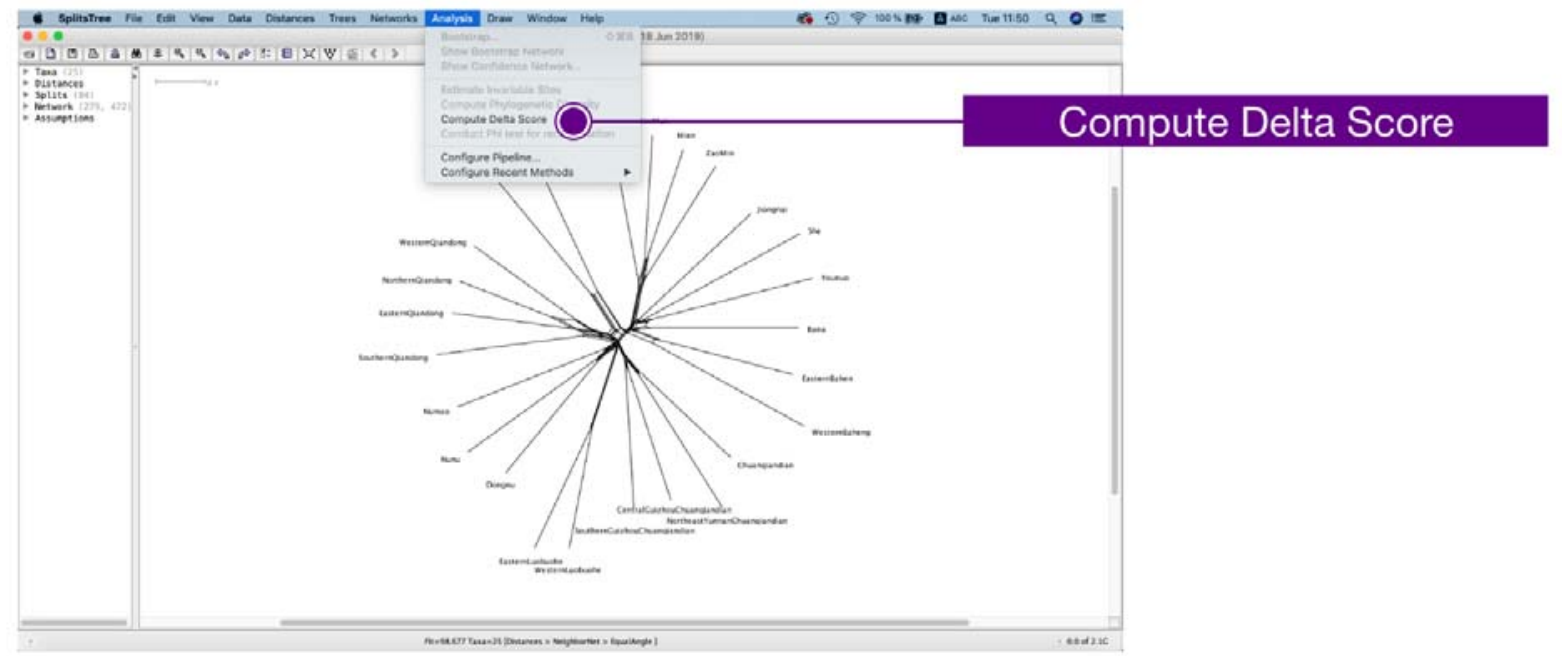

The resulting Neighbor-Net is shown in the following figure. For the purpose of illustration, the Mienic language varieties are colored in red and the Hmongic group is in blue. 


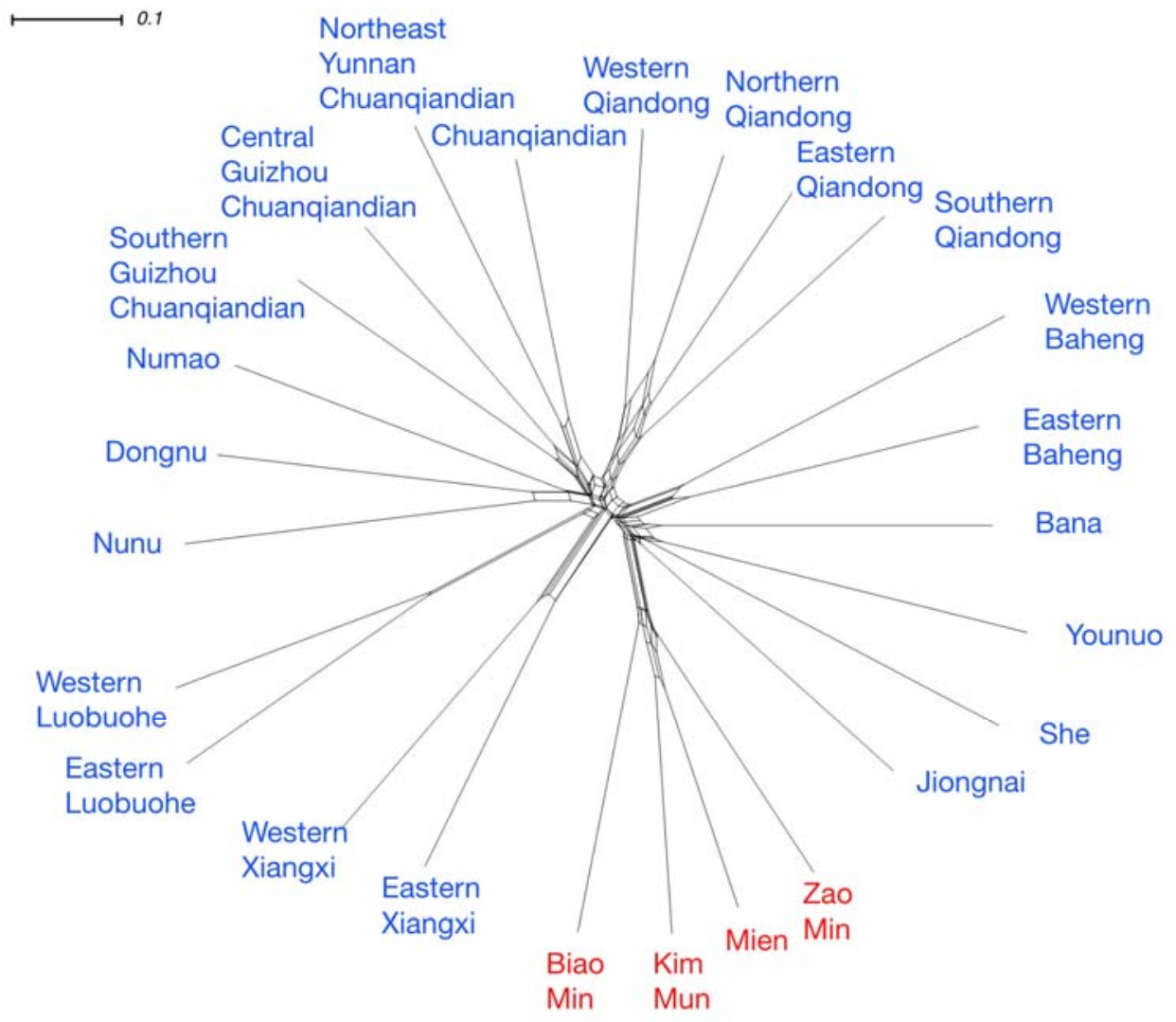

The following table shows the delta scores we computed from the data. 


\begin{tabular}{|c|c|}
\hline Taxon & Delta score \\
\hline Bana & 0.34706 \\
\hline Biao Min & 0.27289 \\
\hline Central Guizhou Chuanqiandian & 0.29924 \\
\hline Chuanqiandian & 0.29172 \\
\hline Dongnu & 0.32416 \\
\hline Eastern Baheng & 0.32056 \\
\hline Eastern Luobuohe & 0.33529 \\
\hline Eastern Qiangong & 0.32083 \\
\hline Eastern Xiangxi & 0.33736 \\
\hline Jiongnai & 0.32644 \\
\hline Kim Mun & 0.26992 \\
\hline Mien & 0.25672 \\
\hline Northeast Yunnan Chanqiandian & 0.29748 \\
\hline Northern Qiandong & 0.28447 \\
\hline Numao & 0.34185 \\
\hline Nunu & 0.32375 \\
\hline She & 0.31671 \\
\hline Southern Guizhou Chuanqiandian & 0.34376 \\
\hline Southern Qiandong & 0.30988 \\
\hline Western Baheng & 0.35259 \\
\hline Western Luobuohe & 0.3211 \\
\hline Western Qiandong & 0.31137 \\
\hline Western Xiangxi & 0.35174 \\
\hline Younuo & 0.2996 \\
\hline Zao Min & 0.26797 \\
\hline
\end{tabular}


The average delta score is 0.313 . As mentioned before, the distances between taxa are calculated via shared cognates. The shorter the distances between two taxa, the higher the similarities between them. If the taxa share cognates not only within their group but also outside their groups, the network finds it challenging to determine the best cluster for them. The larger the reticular structure, or the less tree-like the data is, the higher is the delta score. For one particular language variety's delta score, this means that this specific language contributes to a certain amount of conflict in the data.

\section{Conclusion}

In this tutorial, we provided details of how to execute our workflow for Computer-Assisted Language comparison, using the scripts we wrote, while at the same time illustrating how the results can be manually inspected and modified. We have not discussed the details of the code we wrote, but we recommend users proficient in Python to have a look.

\section{References}

1. List J-M, Walworth M, Greenhill SJ, Tresoldi T, Forkel R. Sequence comparison in computational historical linguistics. Journal of Language Evolution [Internet]. 2018;3(2):130-44. Available from: https://academic.oup.com/jole/article/3/2/130/5050100?guestAccessKey=cf8fe64e-39964cb1-ba2c-317a7cd81bf4

2. 陳其光CQ. Miàoyáo yǔwén [Internet]. Běijīng: Zhōngyāng Mínzú Dàxué 中央民族大学 [Central Institute of Minorities]; 2012. Available from: https://en.wiktionary.org/wiki/Appendix:Hmong-Mien comparative vocabulary list

3. List J-M, Lopez P, Bapteste E. Using sequence similarity networks to identify partial cognates in multilingual wordlists. In: Proceedings of the Association of Computational Linguistics 2016 (Volume 2: Short Papers) [Internet]. Berlin: Association of Computational Linguistics; 2016. pp. 599-605. Available from: http://anthology.aclweb.org/P16-2097

4. Rosvall M, Bergstrom CT. Maps of random walks on complex networks reveal community structure. Proc Natl Acad Sci USA. 2008;105(4):1118-23.

5. List J-M. Automatic inference of sound correspondence patterns across multiple languages. Computational Linguistics [Internet]. 2019;1(45):137-61. Available from: https://www.mitpressjournals.org/doi/full/10.1162/coli a 00344

6. Huson DH. SplitsTree: Analyzing and visualizing evolutionary data. Bioinformatics. 1998;14(1):68-73. 